\title{
A Practical Approach to Dissolution Testing of Type A Medicated Articles
}

\author{
Danna Mattocks ${ }^{1, *}$ and Kailas Thakker ${ }^{2}$ \\ ${ }^{1}$ TherapeuticsMD, 6800 Broken Sound Parkway NW, Boca Raton, FL \\ ${ }^{2}$ Tergus Pharma, 2810 Meridian Parkway, Durham, NC
}

\section{INTRODUCTION}

T he application of dissolution to medicated Type $A$ articles that are combined with animal feed to treat veterinary medical needs is a relatively new application. These products present novel challenges for traditional dissolution methodologies. This article outlines some of the challenges encountered and offers practical solutions for overcoming those challenges.

\section{PURPOSE}

Prior to designing any analytical test, the first question we must ask ourselves is "What is the purpose of the test?" Dissolution is used for many different purposes. Historically, dissolution has been used for formulation development, product comparability, demonstration of manufacturing quality, and support of product shelf life. In the last decade or so, regulatory expectations for dissolution have expanded to include understanding of product critical quality attributes (CQAs), prediction of in vivo performance, and demonstration of clinical relevance.

For medicated feeds, the focus is on using dissolution to support bioequivalence waiver applications for generics. Because this is not a traditional dissolution application, there is very limited literature information available. In addition, the guidance is evolving, and well-established protocols are not available.

While this allows for flexibility in defining the appropriate analytical approach, confidence that the selected approach will be acceptable to the regulators is limited due to lack of prior interactions and successful submission approaches.

\section{PRODUCT ATTRIBUTES}

As illustrated in Table 1, a comparison of product attributes for typical solid oral dose products and medicated Type $A$ articles shows significant differences between the two types of products.

* Corresponding author. e-mail:danna.mattocks@gmail.com

Table 1. Comparison of Product Attributes: Type A Articles vs Solid Oral Dose

\begin{tabular}{|l|l|}
\hline Medicated Feed & Solid Oral Dose \\
\hline Bulk product & Discrete unit product \\
\hline Insoluble excipients & Soluble excipients \\
\hline Dose may be variable & Well-defined dose \\
\hline Aqueous dispersion limited & Easily disperses in aqueous media \\
\hline $\begin{array}{l}\text { Large sample mass typically } \\
\text { measured in g }\end{array}$ & $\begin{array}{l}\text { Small sample mass typically } \\
\text { measured in mg }\end{array}$ \\
\hline
\end{tabular}

\section{Product Attribute Implications}

Dissolution methodology and testing equipment were developed primarily for solid oral dosage forms such as tablets or capsules. While dissolution testing has evolved to include alternate human dosage forms including patches, suspensions, stents, and mediated devices, use for veterinary products is a recent application. Veterinary products have a wider range of attributes than human products, which poses a significant challenge for traditional methodologies.

\section{Sample Size}

Unlike solid oral dose products with discrete unit doses, sample size for medicated Type A articles must often be selected. Determining the appropriate dose to model could depend on multiple factors including species size, average daily intake, and the typical duration of dosing. Due to the complexity, FDA has provided a draft guidance (1) for estimating dosage-adjusted drug solubility based on the wide range of applicable gastric volumes. In addition, the actual quantity consumed per dose may be too large for standard dissolution testing. Typical USP Apparatus 1 and 2 dissolution vessel volumes range from 500 to $1000 \mathrm{~mL}$. Therefore, sample size may be limited by the physical parameters of standard dissolution apparatus options. Is it suitable to assume the dose-to-volume relationship is linear regarding dissolution behavior and scale accordingly? The dose for feed depends on animal weight, drug solubility in gastric volume, and amount consumed and is often calculated as per day average consumption. 


\section{Dispersion Characteristics}

Dispersion of these granular, dry-powder, or particulate products may also significantly influence method parameter selection including media volume and optimal hydrodynamics. For dense products that clump or expand when mixed with aqueous media, these parameters are critical and typical selection criteria may not be applicable.

\section{Sample Introduction}

Due to the dispersion characteristics described above, the technique used for sample introduction into the testing vessel may be critical. For dense samples that clump when wet, simply dropping or pouring the sample can result in a dense clump at the bottom of the vessel that prohibits both adequate wetting of the entire sample and uniform dispersion throughout the vessel. This would in turn give incomplete dissolution and a nonhomogeneous sample solution.

\section{Filtration}

In addition, these products pose unique challenges for filtration. Filtration with typical analytical syringe filters may be impractical for samples with high turbidity due to both soluble and insoluble excipients. Fine particles require small-pore filters. Rapid clogging of the filters due to high particle concentration may result in the need to use an incorporated prefilter layer, multiple filters per sample, or introduction of a prefiltration step through large-pore cannula filters followed by a smaller-pore filter to produce samples that are suitable for UV or HPLC analysis.

\section{Quantitation}

Calculations can be complicated because the product may have units for potency that are different from those of typical pharmaceutical products. However, the analytical reference standards may use typical units especially for antibiotics, which are a common application for Type A medicated articles. As illustrated in the following example, additional complexity may require custom calculations; consequently, typical dissolution calculation templates or validated worksheets will need to be modified.

\section{Example Type A Product}

- Product Label Claim = grams active $/$ pound

- Standard (std) concentration = units of activity/ gram $\times$ std wt $(\mathrm{g}) / \mathrm{std}$ vol $(\mathrm{mL})=$ units of activity/ $\mathrm{mL}$

- Sample (spl) content $=$ label claim $(\mathrm{g} / \mathrm{lb}) \times$ $\mathrm{lb} / 453.59 \mathrm{~g} \times \mathrm{spl} w \mathrm{wt}(\mathrm{g}) \times$ units of activity $/ \mathrm{g}=$ units of activity

- $\%$ Dissolved $=$ sample content (activity)/sample vol $(\mathrm{mL}) /$ standard concentration (activity $/ \mathrm{mL}$ ) $\times 100 \%$
The bottom line is that the analyst must ensure calculations accurately represent reported sample strength and standard concentration.

\section{USP Apparatus 1 and 2 Test Design}

Method development for Type A medicated articles requires a flexible approach that takes both the product and test equipment attributes into consideration. As outlined earlier, parameters such as product dose and sample size, which are clearly defined with no flexibility for solid oral dosage forms, must be determined for these products.

\section{Sample Considerations}

Determining the actual dose for these products can be a challenge. It is important to remember that medicated Type $A$ articles are not consumed by the animals directly. The product to be tested is added to animal feed by a variety of methods, and the feed is then consumed. While daily average consumption is used to calculate the amount of Type A article that should be added to the feed, the actual consumption, especially by sick animals, is highly variable.

In any case, for dissolution studies using currently available test equipment, the in vitro limitations must be considered as well as the in vivo consumption. An analytical sample size reflecting the actual dose consumed will generally not be feasible, with poultry being the possible exception. Solubility of the active component and, to a lesser degree, of the soluble excipients will determine maximum sample size.

\section{System Considerations}

Regardless of theoretical dose, the vessel volume and basket capacity are likely to be the overriding practical limitations to sample size for Apparatus 1 and 2 testing. In addition, hydrodynamics may be paramount in achieving reproducible results for products with poor dispersion characteristics.

\section{Volume}

A basic assumption for dissolution methods is that sink conditions will be achieved. The accepted definition of sink conditions is a minimum of three times the equilibrium solubility of the active component at full release for a single dosage unit.

While surfactants can be expected to increase solubility limits, their effectiveness may be limited by the physical characteristics of Type A medicated articles as outlined previously. If the product clumps in aqueous media, has poor dispersion, or has limited wettability, then the 
ability of the surfactant to pull the active into solution may be inhibited. In addition, solubility can be negatively impacted by the presence of solubilized excipients in the medium.

As illustrated in Table 2, the gastric volume for large species will be impossible to replicate, and for small species, a modified low-volume system would be required (2).

Table 2. Values for Estimating Drug Solubility Based Upon "Dosage Adjusted" Method ${ }^{a, b}, c$ (Reprinted from ref 2.)

\begin{tabular}{|c|c|c|}
\hline Species & $\begin{array}{c}\text { Body weight } \\
\text { (for estimating drug } \\
\text { intake) }\end{array}$ & $\begin{array}{c}\text { "Gastric" volume } \\
\text { (to be used as volume } \\
\text { of solvent) }\end{array}$ \\
\hline Cattle & $400 \mathrm{~kg}$ & $200 \mathrm{~L}$ (rumen) \\
\hline Pre-ruminating calf & $60 \mathrm{~kg}$ & $2 \mathrm{~L}$ \\
\hline Swine & $200 \mathrm{~kg}$ & $8 \mathrm{~L}$ \\
\hline Horse & $450 \mathrm{~kg}$ & $18 \mathrm{~L}$ \\
\hline Chicken & $2.5 \mathrm{~kg}$ & $0.1 \mathrm{~L}$ \\
\hline Turkey & $10 \mathrm{~kg}$ & $0.4 \mathrm{~L}$ \\
\hline
\end{tabular}

${ }^{a}$ Gastric volume estimate for cattle, swine, and horses are based upon values reported in ME Ensminger, JE Oldfield, and WW Heinemann, Feeds and Nutrition, 1990, Ensminter Publishing Company, CA, p 53.

bVolume, based upon daily water consumption, is based upon values reported in GD Goldstein and E Skadhauge, Renal and extrarenal regulation of body fluid composition, In: Avian Physiology, 2000, GC Whittow, ed., Academic Press, FL, pp 265-291 and Average Daily and Annual Water Requirements, Government of Alberta, Canada, http://www1.agric.gov. ab.ca/app19/calc/livestock/waterreg_dataentry2.jsp.

'Animal weights are based upon the following references: swine (NRC, Nutrient Requirements of Swine 1988, poultry (NRC, Nutrient Requirements of Poultry 1994), and beef cattle (NRC, Nutrient Requirements of Beef Cattle 1984).

\section{PRACTICAL APPLICATION}

For USP Apparatus 1 and 2 methods, sample size is limited by available medium volume. To determine appropriate sample size, determine the target concentration at full release and ensure that sink conditions are maintained.

Product active content is based on larger quantities with wider specification ranges than typical human pharmaceutical products. A smaller sample size necessitated by instrument limitations may not be uniform or representative of the product batch. Consequently, nominal sample content correction based on assay determination or wider specifications may be required.

Type A medicated articles may have variable granule/ particle size within a sample. Pre dissolution prep may be required for large particles to achieve adequate active content and uniformity for sample sizes that meet the volume and basket limitations. Variable particle size may impact sample-to-sample reproducibility for both intact and prepared samples. Grinding is an option for pre-dissolution sample prep; however, dissolution is traditionally performed on intact product, so this approach may not meet agency expectations.

\section{KEY CONSIDERATIONS FOR APPARATUS SELECTION}

\section{Apparatus 2}

- Dense material that rapidly sinks to the bottom of the vessel may experience significant coning in Apparatus 2 systems.

- Wettability or clumping of sample resulting in inadequate medium exposure may limit active release even with high paddle speeds.

- Sample introduction is a challenge that requires significant stagger; automated systems may not be suitable.

- Optimize paddle speed to achieve a gradual profile reaching $\geq 85 \%$ released.

- If less than $85 \%$ is released, reduce sample size and perform infinity test to determine if low release is due to a solubility limitation or is a hydrodynamic artifact.

- Assay testing of proposed sample aliquot is recommended if less than full release is observed even with infinity test.

\section{Apparatus 1}

- Use of baskets may help uniformly disperse sample and increase medium exposure.

- Use of baskets limits sample size to no more than basket volume and generally less due to clumping and wetting issues if the basket is filled to volume.

- Basket mesh size change can address some sample clumping/wetting issues.

- Sample dispersion from baskets can reduce initial clumping.

- Small-mesh baskets may result in significant clumping within the baskets.

- Large-mesh baskets may help reduce sample clumping both within the baskets and in the bottom of the vessel.

- Sample introduction is a challenge if particles are smaller than the mesh openings because loss of sample will occur prior to test start. 


\section{CONCLUSION}

Type A medicated articles have many characteristics and key considerations related to the sample that are not found in human solid oral dose products. These challenges may require flexible approaches to method development.

- Use of Apparatus 1 and 2 can deliver suitable profiles for these products.

- Discrimination for $\mathrm{pH}$, sample size, rotation speed, and basket mesh size can be obtained.

- Sample uniformity for small sample size may be an issue.

- Higher variability than for typical solid oral dose product may be observed.

Dissolution testing of Type A medicated articles is complex, and typical development strategies may not be suitable. However, Apparatus 1 and 2 method development resulting in a gradual profile with typical specification limits can be successfully executed.

\section{REFERENCES}

1. Modified Release Veterinary Parenteral Dosage Forms: Development, Evaluation, and Establishment of Specifications; Guidance for Industry; U.S. Department of Health and Human Services, Food and Drug Administration, Center for Veterinary Medicine (FDA-CVM), U.S. Government Printing Office: Washington, DC, 2016.

2. Waivers of In Vivo Demonstration of Bioequivalence of Animal Drugs in Soluble Powder Oral Dosage Form Products and Type A Medicated Articles; Guidance for Industry; U.S. Department of Health and Human Services, Food and Drug Administration, Center for Veterinary Medicine (FDA-CVM), U.S. Government Printing Office: Washington, DC, 2004. 